\title{
A Superconvergent Finite Element Method for the Korteweg-de Vries Equation
}

\author{
By Douglas N. Arnold* and Ragnar Winther
}

\begin{abstract}
An unconditionally stable fully discrete finite element method for the Korteweg-de Vries equation is presented. In addition to satisfying optimal order global estimates, it is shown that this method is superconvergent at the nodes. The algorithm is derived from the conservative method proposed by the second author by the introduction of a small time-independent forcing term into the discrete equations. This term is a form of the quasiprojection which was first employed in the analysis of superconvergence phenomena for parabolic problems. However, in the present work, unlike in the parabolic case, the quasiprojection is used as perturbation of the discrete equations and does not affect the choice of initial values.
\end{abstract}

1. Introduction. The Korteweg-de Vries equation arises in the modelling of long unidirectional water waves of small amplitude and similar phenomena. See [6] for a discussion. We shall consider the periodic initial value problem. Thus, given a smooth 1-periodic function $u_{0}$, the solution $u(x, t)$ is defined by

$$
\begin{array}{ll}
u_{t}+2 u u_{x}+u_{x x x}=0, & x \in \mathbf{R}, 0 \leqslant t \leqslant T, \\
u(x, 0)=u_{0}(x), & x \in \mathbf{R}, \\
u(x, t)=u(x+1, t), & x \in \mathbf{R}, 0 \leqslant t \leqslant T .
\end{array}
$$

In [7] the second author presented a finite element procedure for approximating the solution. In this procedure spatial discretization is accomplished by a Galerkin method with the trial and test spaces consisting of piecewise polynomials of differing degrees. Timestepping is by an unconditionally stable three-level scheme which requires the factorization of only a single periodic band matrix once for all timesteps. It was shown that as the mesh is refined the approximation converges in the $L^{2}$ and $H^{1}$ norms at the optimal asymptotic rate for the degree of the trial functions employed.

In this paper we introduce a related finite element method which retains these desirable properties and is, in addition, superconvergent. This means that as the mesh is refined the function values at the nodes of the partition converge with an asymptotic rate roughly equal to twice the global rate. The present method is distinguished from that of [7] by the presence of a particular small time-independent forcing term in the discrete equations. This term is closely related to the quasiprojection introduced by Douglas, Dupont, and Wheeler in their paper [5]

Received September 8, 1980; revised April 1, 1981.

1980 Mathematics Subject Classification. Primary 65N30, 65N15; Secondary 76B15.

* The work of this author was supported in part by the General Research Board of the University of Maryland. 
establishing superconvergence for finite element methods applied to linear secondorder parabolic and hyperbolic problems, and generalized to nonlinear parabolic problems in [1]. However, unlike in these works, we do not use our quasiprojection to alter the initialization of the method. Rather the defining equations themselves are altered.

The extra computational work involved in the present scheme as compared to that of [7] is small and does not increase with the number of timesteps, although additional code is required. The authors feel that the present scheme supercedes the earlier one at least whenever high accuracy is desired. The implementation of the scheme is summarized in Section 5 of the paper.

An alternative to the Korteweg-de Vries equation has been proposed in [3]. Superconvergence of a standard finite element method for this and other Sobolev equations has been established by related but simpler means and without the use of a quasiprojection [2].

2. Notations and Preliminaries. The $j$ th derivative with respect to $x$ of a function $f$ will of ten be denoted $d_{x}^{j} f$, and $d_{x}^{1} f$ will be abbreviated to $d_{x} f$. Similarly, $d_{x}^{j} f$ denotes the $j$ th derivative with respect to the temporal variable. For small $j$ the subscript notation will also be used: $f_{x}, f_{x x}, f_{x t}$, etc.

If $J$ is an interval and $m$ a nonnegative integer, $H^{m}(J)$ shall denote the usual Sobolev space. When $J$ is omitted the unit interval $I$ is assumed. The addition of the subscript $\mathfrak{p}$ indicates periodicity:

$$
H_{\mathfrak{p}}^{m}=\left\{f \in H^{m}(I)\left|d_{x}^{j} f\right|_{0}^{1}=0, j=0,1, \ldots, m-1\right\} .
$$

The norm on this space is the usual $H^{m}$ norm and will be denoted by $\|\cdot\|_{m}$, or simply $\|\cdot\|$ if $m=0$. The dual norm to $H_{\mathfrak{b}}^{m}$ with respect to the $H^{0}$ inner product is denoted $\|\cdot\|_{-m}$. Each of these spaces is a Hilbert space and, for $m$ positive, a Banach algebra.

Bona and Smith [4] have established regularity results in these spaces for the equation (1.1). In particular, if $u_{0}$ is a smooth periodic function, then $u$ is smooth and there is a constant $C$, depending only on $\left\|u_{0}\right\|_{s}$ and $s$ such that

$$
\left\|d_{t}^{j} u\right\|_{s-3 j} \leqslant C, \quad 0 \leqslant t \leqslant T,
$$

for integers $s \geqslant 2$ and $j \geqslant 0$ satisfying $s-3 j \geqslant 0$. This is shown in the proof of Theorem 3 of [4] for the pure initial value problem and $s \geqslant 3$. The case $s=2$ and that of periodicity conditions are covered in the appendices.

We let $(\phi, \psi)=\int_{0}^{1} \phi(x) \psi(x) d x$ denote the inner product in $H^{0}$. If $S$ is any subspace of $H^{0}$, then

$$
\hat{S}=\{f \in S \mid(f, 1)=0\}
$$

is the closed subspace consisting of functions with mean value zero. Note that on the space $\hat{H}_{\mathfrak{p}}^{1}$ the inner product $A(\phi, \psi)=\left(\phi_{x}, \psi_{x}\right)$ gives rise to a norm equivalent to the $H^{1}$ norm.

A bounded linear operator $\Lambda: H^{0} \rightarrow \hat{H}_{\mathfrak{p}}^{1}$ is defined by $(\Lambda \phi)_{x}=\phi-(\phi, 1)$. Note that $\Lambda$ maps $H_{\mathfrak{p}}^{m-1}$ boundedly into $\hat{H}_{\mathfrak{p}}^{m}$ for $m \geqslant 0$. In fact $\phi \mapsto\|\Lambda \phi\|_{m}+|(\phi, 1)|$ is 
equivalent to the $m-1$ norm. Also $\Lambda 1=0$ and $\Lambda \phi_{x}=\phi-(\phi, 1)$. Moreover, for $\phi, \psi \in H^{0}$

$$
(\Lambda \phi, \psi)=(\Lambda \phi, \psi-(\psi, 1))=\left(\Lambda \phi,(\Lambda \psi)_{x}\right)
$$

and hence $\Lambda$ is antisymmetric on $H^{0}$.

The finite element spaces are defined with reference to partitions $\delta=\left\{x_{i}\right\}_{i=0}^{M}$, where $M$ is a positive integer depending on $\delta$, and $0=x_{0}<x_{1}<\cdots<x_{M}=1$. For a fixed integer $r \geqslant 2$, let $\mathscr{P}_{r}(\delta)$ be the space of all continuous functions on $I$ whose restriction to each interval $\left[x_{i-1}, x_{i}\right]$ coincides with a polynomial of degree less than $r$. The trial and test spaces we shall use are, respectively,

$$
S=S_{\delta}=H_{\mathfrak{p}}^{1} \cap \mathscr{P}_{r}(\delta) \quad \text { and } \quad S^{*}=S_{\delta}^{*}=H_{\mathfrak{p}}^{2} \cap \mathscr{P}_{r+1}(\delta) .
$$

Note that $\operatorname{dim} S=\operatorname{dim} S^{*}=M(r-1)$.

An elliptic projector $P_{1}: H_{\mathfrak{p}}^{1} \rightarrow S$ is defined by $A\left(P_{1} \phi, \psi\right)=A(\phi, \psi)$ for all $\psi \in \hat{S}$ and $\left(P_{1} \phi, 1\right)=(\phi, 1)$. We set $V=P_{1} u$. We also let $P_{0}: L^{2} \rightarrow S$ denote the $L^{2}$ projection.

Let $h=h_{\delta}=\max \left(x_{i}-x_{i-1}\right)$. It is well known that there is a constant $C$ such that

$$
\left\|P_{1} w-w\right\|+h\left\|P_{1} w-w\right\|_{1} \leqslant C h^{s}\|w\|_{s}, \quad w \in H^{s}, 1 \leqslant s<r .
$$

In addition, we assume that, for some constant $C,\left\|P_{0} w\right\|_{1}<C\|w\|_{1}$ for all partitions under consideration. This is the case if we restrict ourselves to a quasiuniform family of partitions. It implies that

$$
\|\phi\|_{-1} \leqslant C \sup \left\{(\phi, \psi) \mid \psi \in S,\|\psi\|_{1}=1\right\}, \quad \phi \in S .
$$

Let $N$ denote the number of timesteps and $k=T / N$ the timestep. We shall introduce numerous functions $W$ defined on a subset of $\{0,1, \ldots, N\}$ and taking values $W^{n} \in H^{0}$. In particular with each function $f:[0, T] \rightarrow H^{0}$ we have an associated function defined by $f^{n}=f(n k)$. When $f$ is $C^{2}$ we also define an approximation $\bar{f}$ to $f^{1}$ by

$$
\bar{f}=f^{0}+k f_{t}^{0}+k^{2} f_{t t}^{0} / 2 .
$$

If $W$ is defined on $\{0,1, \ldots, N\}$, then we define $D_{+} W, D W$, and $\tilde{W}$ by

$$
\begin{aligned}
D_{+} W^{n} & =\left(W^{n+1}-W^{n}\right) / k, & & n=0,1, \ldots, N-1, \\
D W^{n} & =\left(W^{n+1}-W^{n-1}\right) / 2 k, & & n=1,2, \ldots, N-1, \\
\tilde{W}^{n} & =\left(W^{n+1}+W^{n-1}\right) / 2, & & n=1,2, \ldots, N-1 .
\end{aligned}
$$

Having set forth these notations, we define the fully discrete finite element solution to the Korteweg-de Vries equation as the function $U:\{0,1, \ldots, N\} \rightarrow S$ satisfying

$$
(D U, \chi)-\left((U)^{2}, \chi_{x}\right)+\left(\tilde{U}_{x}, \chi_{x x}\right)=\left(\varepsilon, \chi_{x}\right)
$$

$$
\chi \in S^{*}, n=1,2, \ldots, N-1 .
$$

Here the perturbation $\varepsilon \in \hat{S}$ and the initial functions $U^{0}$ and $U^{1}$ have to be specified. We shall always assume that $\varepsilon$ is independent of $n$ and satisfies

$$
\|\varepsilon\|_{-1} \leqslant C\left\{k^{2}+h^{r-1}\right\} \text {. }
$$


In the case $\varepsilon \equiv 0$, this method was analyzed in [7] and optimal order $H^{1}$ and $H^{0}$ error estimates derived. Note that once $\varepsilon, U^{0}$, and $U^{1}$ are given, the determination of $U$ requires only the factorization of a single periodic band matrix, once for all timesteps.

Observe that the mean value of the solution $u$ of (1.1) is independent of time. If $U^{0}$ and $U^{1}$ are specified such that $\left(U^{0}, 1\right)=\left(U^{1}, 1\right)=\left(u_{0}, 1\right)$, as they will be, then the method (2.3) is equivalent to determining $U:\{0,1, \ldots, N\} \rightarrow \hat{S}+\left(u_{0}, 1\right)=$ $\left\{W \in S \mid(W, 1)=\left(u_{0}, 1\right)\right\}$ such that

$$
-\left(\Lambda D U+(U)^{2}, \mu\right)+A(\tilde{U}, \mu)=(\varepsilon, \mu), \quad \mu \in \hat{S}, n=1,2, \ldots, N-1 .
$$

Assume that $\varepsilon \in \hat{S}$ is determined by

$$
(\varepsilon, \mu)=-\left(\Lambda W+\left(U^{1}\right)^{2}, \mu\right)+A\left(U^{0}+k W, \mu\right), \quad \mu \in \hat{S},
$$

for a given $W \in \hat{S}$. Then (2.5) implies that

$$
D U^{1}=W \text {. }
$$

In this way the perturbation $\varepsilon$ serves to impose a specific value for $D U^{1}$. This will be used later to guarantee that $\left\|D\left(u^{n}-U^{n}\right)\right\|_{1}$ is sufficiently small. Let us remark in passing that the technique of perturbing the discrete equations to force the initial value of a time difference or derivative is applicable more generally.

Now, let $e=u-U$ denote the error. The $H^{1}$ convergence estimates for the method (2.3) are stated below. These estimates generalize those of Theorem 5.1 in [7] and may be obtained by an argument similar to the one in [7] by considering the perturbation $\varepsilon$ as an additional error term.

TheOREM 2.1. Assume that the perturbation $\varepsilon$ satisfies (2.4). Then there is a positive constant $C$, depending on $\left\|u_{0}\right\|_{r+9}$, such that if $h \leqslant C^{-1}$ and $\left\|e^{0}\right\|_{1},\left\|e^{1}\right\|_{1},\left\|D_{+} e^{0}\right\|_{1}$, and $\left\|D_{+} e^{1}\right\|_{1} \leqslant C$, then the error in the solution $U$ of (2.3) satisfies

$$
\left\|e^{n}\right\|_{1} \leqslant C\left(k^{2}+h^{r-1}+\left\|e^{0}\right\|_{1}+\left\|e^{1}\right\|_{1}\right), \quad n=0,1, \ldots, N,
$$

and

$$
\begin{aligned}
\left\|D_{+} e^{n}\right\|_{1} \leqslant C\left(k^{2}+h^{r-1}+\left\|e^{0}\right\|_{1}+\left\|e^{1}\right\|_{1}+\left\|D_{+} e^{0}\right\|_{1}+\left\|D_{+} e^{1}\right\|_{1}\right) & \\
n & =0,1, \ldots, N-1 .
\end{aligned}
$$

Note that Theorem 2.1 does not provide error estimates in terms solely of known quantities, because $D_{+} e^{1}$ is not known a priori. This difficulty may be overcome by proper choice of the perturbation $\varepsilon$ as indicated in the previous paragraph. In Section 4 we shall make particular choices of $U^{0}, U^{1}$, and $\varepsilon$. It should be remarked that the methods of [7] may also be used to prove optimal order error estimates in $H^{0}$, but we shall not need these.

3. The Superconvergent Perturbation Method. In this section we specify the perturbation function $\varepsilon$ and the initial values and state the superconvergence theorem. To motivate our choice of $\varepsilon$, we consider first a semidiscrete finite element method for the linear equation $u_{t}+u_{x}+u_{x x x}=0$ and outline in this simpler context the main ideas leading to the superconvergence estimate. The fully discrete nonlinear case will be treated in full detail in the following section. 
The exact solution $u:[0, T] \rightarrow H_{\mathfrak{p}}^{1}$ to the linear equation is determined by its mean value and the equations

$$
(J u, \mu)-A(u, \mu)=0, \quad \mu \in H_{\mathfrak{p}}^{1}, 0 \leqslant t \leqslant T,
$$

where $J u=\Lambda u_{t}+u$. The approximate solution $U:[0, T] \rightarrow \hat{S}+\left(u_{0}, 1\right)$ is defined by the system of ordinary differential equations

$$
(J U, \mu)-A(U, \mu)=-(\varepsilon, \mu), \quad \mu \in \hat{S}, 0 \leqslant t<T .
$$

The perturbation $\varepsilon \in \hat{S}$ will be specified below. The initial value of $U$ is taken to be $V(0)$ (for the time being $V$ denotes the elliptic projection of the solution of the linear equation). In order to be sure that the discretized system has a solution, we assume that $P_{0} \Lambda \hat{S}=\hat{S}$, or, equivalently, that $P_{0} S^{*}=S$. However, no such assumption will be necessary when we consider the fully discrete method.

Our goal is that the error $e=u-U$ satisfy in addition to the global estimates

$$
\|e\|_{1}+\left\|e_{t}\right\|_{1} \leqslant C h^{r-1}, \quad 0 \leqslant t \leqslant T,
$$

also the superconvergent estimate

$$
|e(\bar{x}, t)| \leqslant C h^{2(r-1)}, \quad \bar{x} \in \delta, 0 \leqslant t \leqslant T .
$$

We begin by comparing $U$ to $V$. Letting $Z_{0}=u-V, \theta_{0}=V-U$, we have

$$
(J V, \mu)-A(V, \mu)=-\left(J Z_{0}, \mu\right), \quad \mu \in \hat{S}, 0 \leqslant t<T,
$$

so

$$
\left(J \theta_{0}, \mu\right)-A\left(\theta_{0}, \mu\right)=-\left(J Z_{0}-\varepsilon, \mu\right), \quad \mu \in \hat{S}, 0 \leqslant t \leqslant T .
$$

The quasiprojection is a sum whose terms, $Z_{i}:[0, T] \rightarrow \hat{S}$, are inductively defined by the relation

$$
A\left(Z_{i}, \mu\right)=\left(J Z_{i-1}-J\left(Z_{i-1}\right)(0), \mu\right), \quad \mu \in \hat{S}, 0 \leqslant t \leqslant T, i=1,2, \ldots
$$

From this definition follow four essential consequences, which will be proved in the nonlinear case. First, setting $t=0$, we have $Z_{i}(0)=0$ for $i=1,2, \ldots$ Second, the successive terms decrease in size. In particular, for $j=[(r-1) / 2]$

$$
\left\|Z_{j}\right\|+\left\|d_{t} Z_{j}\right\| \leqslant C h^{2(r-1)}, \quad 0 \leqslant t \leqslant T .
$$

Third, using the techniques available for analyzing superconvergence for elliptic problems in one dimension, one can show that

$$
\left|Z_{i}(\bar{x}, t)\right| \leqslant C h^{2(r-1)}, \quad \bar{x} \in \delta, 0 \leqslant t \leqslant T, i=0,1, \ldots, j .
$$

Finally, the definition of the quasiprojection is contrived so that if we substitute $\sum_{i=1}^{j} Z_{i}$ into the form associated with the linear differential operator, the timedependent terms form a telescoping sum:

$$
\begin{aligned}
& \sum_{i=1}^{j}\left[\left(J Z_{i}, \mu\right)-A\left(Z_{i}, \mu\right)\right] \\
& \quad=\left(J\left(Z_{j}-Z_{0}\right)+J\left(\sum_{i=0}^{j-1} Z_{i}\right)(0), \mu\right), \quad \mu \in \hat{S}, 0 \leqslant t \leqslant T .
\end{aligned}
$$


Therefore, setting $\theta=\theta_{0}-\sum_{i=1}^{j} Z_{i}$, we have $\theta(0)=0$ and

$$
(J \theta, \mu)-A(\theta, \mu)=-\left(J Z_{j}+J\left(\sum_{i=0}^{j-1} Z_{i}\right)(0)-\varepsilon, \mu\right), \quad \mu \in \hat{S}, 0<t<T .
$$

Now, $e=\theta+\sum_{i=0}^{j} Z_{i}$, and so, in view of (3.4), to establish (3.2) it suffices to show that $\|\theta\|_{1} \leqslant C h^{2(r-1)}$ for all $t$. This may be accomplished by the method of energy estimates if the quantity $J Z_{j}+J\left(\sum_{i=0}^{j-1} Z_{i}\right)(0)-\varepsilon$, appearing on the righthand side of (3.5), is of order $h^{2(r-1)}$ in $H^{0}$. In light of (3.3) this is so if and only if $\varepsilon-J\left(\sum_{i=0}^{j-1} Z_{i}\right)(0)$ is of this order. A particularly convenient choice is $\varepsilon=$ $J\left(\sum_{i=0}^{j} Z_{i}\right)(0)$. With this choice $(3.5)$ becomes

$$
(J \theta, \mu)-A(\theta, \mu)=-\left(J Z_{j}-J\left(Z_{j}\right)(0), \mu\right), \quad \mu \in \hat{S}, 0 \leqslant t \leqslant T .
$$

It then follows that $\theta_{t}(0)=0$. This enables us to show that $\left\|e_{t}(0)\right\|_{1}<C h^{r-1}$, and thus to obtain the optimal order estimate on $e_{t}$ stated in (3.1) by an analogue of Theorem 2.1. Such an estimate is essential in the nonlinear case.

Returning now to the Korteweg-de Vries equation, we define the fully discrete finite element method which will be analyzed in the next section. The definitions of the quasiprojection and perturbation we now offer will remain in effect for the remainder of the paper. These are similar to the definitions given above but are complicated by the nonlinearity and time discretization.

Let $Z_{0}=u-V$. For $i=1,2, \ldots, j=[(r-1) / 2]$ define $Z_{i}:[0, T] \rightarrow \hat{S}$ by

$$
\begin{aligned}
A\left(Z_{i}, \mu\right)=\left(\left[\Lambda d_{t}+2 u\right] Z_{i-1}-\left[\Lambda \overline{d_{t} Z_{i-1}}+2 \bar{u} \bar{Z}_{i-1}\right], \mu\right) & \\
\mu & \in \hat{S}, 0<t \leqslant T .
\end{aligned}
$$

Recall that the bar notation represents an extrapolation to $t=k$ defined in (2.2). We now determine our finite element method by fixing values for $U^{0}, U^{1}$, and $\varepsilon$. Let $\varepsilon \in \hat{S}$ be defined by (2.6) with

$$
W=\bar{V}_{t}-\sum_{i=1}^{j} \overline{d_{t} Z_{i}}
$$

Choosing initial values $U^{0}=V^{0}, U^{1}=\bar{V}$, the approximate solution is then defined by (2.3) or, equivalently, (2.5).

By (1.1) and the definitions of $U^{0}$ and $V$, we have for all $\mu \in S$ that

$$
\begin{aligned}
A\left(U^{0}+k \bar{V}_{t}, \mu\right) & =A\left(u^{0}+k \bar{u}_{t}, \mu\right)=A\left(u^{1}, \mu\right)-\left(d_{x}^{2}\left(u^{0}+k \bar{u}_{t}-u^{1}\right), \mu\right) \\
& =\left(\Lambda u_{t}^{1}+\left(u^{1}\right)^{2}, \mu\right)-\left(d_{x}^{2}\left(u^{0}+k \bar{u}_{t}-u^{1}\right), \mu\right)
\end{aligned}
$$

Hence,

$$
\begin{aligned}
-\left(\Lambda \bar{V}_{t}+\left(U^{1}\right)^{2}, \mu\right)+A\left(U^{0}+k \bar{V}_{t}, \mu\right) & \\
= & \left(\Lambda\left(\bar{u}_{t}-\bar{V}_{t}\right)+\Lambda\left(u_{t}^{1}-\bar{u}_{t}\right)+\left(u^{1}\right)^{2}-\left(U^{1}\right)^{2}-d_{x}^{2}\left(u^{0}+k \bar{u}_{t}-u^{1}\right), \mu\right) \\
= & \left(\Lambda \overline{d_{t} Z_{0}}+2 u^{1} \bar{Z}_{0}+2 u^{1}\left(u^{1}-\bar{u}\right)+\Lambda\left(u_{t}^{1}-\bar{u}_{t}\right)\right. \\
& \left.-d_{x}^{2}\left(u^{0}+k u_{t}-u^{1}\right)-\left(e^{1}\right)^{2}, \mu\right) .
\end{aligned}
$$


Therefore, from (2.6),

$$
\begin{aligned}
(\varepsilon, \mu)= & \left(\Lambda \sum_{i=0}^{j} \overline{d_{t} Z_{i}}+2 u^{1} \bar{Z}_{0}+2 u^{1}\left(u^{1}-\bar{u}\right)\right. \\
& \left.\quad+\Lambda\left(u_{t}^{1}-\bar{u}_{t}\right)-d_{x}^{2}\left(u^{0}+k \bar{u}_{t}-u^{1}\right)-\left(e^{1}\right)^{2}, \mu\right)-k \sum_{i=1}^{j} A\left(\overline{d_{t} Z_{i}}, \mu\right) \\
& =\sum_{i=0}^{j}\left(\Lambda \overline{d_{t} Z_{i}}+2 \bar{u} \bar{Z}_{i}, \mu\right) \\
& +\left(2\left(u^{1}+\bar{Z}_{0}\right)\left(u^{1}-\bar{u}\right)-2 \bar{u} \sum_{i=1}^{j} \bar{Z}_{i}+\Lambda\left(u_{t}^{1}-\bar{u}_{t}\right)\right. \\
& -k \sum_{i=1}^{j} A\left(\bar{d}_{t} Z_{i}, \mu\right), \quad \mu \in S .
\end{aligned}
$$

In the next section we will show that

$$
\|\varepsilon\|_{-1}+\left\|e^{0}\right\|_{1}+\left\|e^{1}\right\|_{1}+\left\|D_{+} e^{0}\right\|_{1}+\left\|D_{+} e^{1}\right\|_{1} \leqslant C\left(k^{2}+h^{r-1}\right),
$$

and hence, by Theorem 2.1, we have the global estimates

$$
\max _{0<n<N}\left\|e^{n}\right\|_{1}+\max _{0<n<N-1}\left\|D_{+} e^{n}\right\|_{1} \leqslant C\left(k^{2}+h^{r-1}\right) .
$$

The major result of this paper is the following theorem, which is proved in the next section.

SuPERCONVERgence TheOREM. There exists a positive constant $C$ (depending on $\left.\left\|u_{0}\right\|_{r+3 j+9}\right)$ such that if $h \leqslant C^{-1}$, then

$$
\left|e^{n}(\bar{x})\right| \leqslant C\left(k^{2}+h^{2(r-1)}\right), \quad \bar{x} \in \delta, n=0,1, \ldots, N .
$$

4. Proof of the Superconvergence Theorem. The first step of the proof is the estimation of the terms of the quasiprojection. As is often the case in analyses of superconvergence, an appropriately constructed family of negative-order Sobolev norms is essential. Following [5], [1], we fix a particular knot $\bar{x} \in \delta$ and define for positive integral $s$

$$
\tilde{H}^{s}=H_{\mathfrak{p}}^{1} \cap H^{s}((\bar{x}, \bar{x}+1)) .
$$

The norm on this Hilbert space is the restriction of the usual norm on $H^{s}((\bar{x}, \bar{x}+1))$ and will be denoted $\|\cdot\|_{s}$. Note that $\tilde{H}^{1}=H_{\mathfrak{p}}^{1}$ with the usual norm. Since $\bar{x}$ is a node, the functions in $S$ approximate functions in $\tilde{H}^{s}$ well. Specifically, there is a constant $C$ such that

$$
\inf _{\mu \in S}\|\phi-\mu\|_{1} \leqslant C h^{s-1}\|\| \phi \|_{s}, \quad \phi \in \tilde{H}^{s}, 1 \leqslant s \leqslant r .
$$

LeMMA 4.1. Given $\rho \in H^{0}$, there exists a unique function $\phi \in \hat{H}_{\mathfrak{p}}^{2}$ such that

$$
A(\psi, \phi)=(\psi, \rho), \quad \psi \in \hat{H}^{1} .
$$


Moreover, if $\rho \in \tilde{H}^{s}$, then $\phi \in \tilde{H}^{s+2}$, and there exists a constant $C$, independent of $\rho$ and $\bar{x}$, such that

$$
\|\phi \mid\|_{s+2} \leqslant C\|\rho\|_{s}
$$

Proof. By the Riesz representation theorem there is a unique element $\phi$ of $\hat{H}_{\mathfrak{b}}^{1}$ satisfying (4.1). It is easy to see that $\phi$ is the solution in $\hat{H}_{\mathfrak{p}}^{2}$ of the differential equation

$$
-\phi_{x x}=\rho-(\rho, 1) \text {. }
$$

Since $|\phi(\bar{x})| \leqslant\left\|\phi_{x}\right\| \leqslant\|\rho\| \leqslant\|\rho\|_{s}$, regularity of the Dirichlet two-point boundary value problem implies (4.2).

Next define for each $Z \in H^{1}$ and each nonnegative integer $s$

$$
\|Z\|_{-s}=\sup \left\{A(Z, \phi) \mid \phi \in\left(\tilde{H}^{s+2}\right)^{\wedge},\|\phi\|_{s+2}=1\right\} .
$$

The importance of these norms depends on the fact, proven below, that $\|Z \mid\|_{-s}$ dominates the nodal value $Z(\bar{x})$ for $Z \in \hat{H}_{\mathfrak{p}}^{1}$. This property is of course not possessed by the more familiar norms $\|Z\|_{-s}$.

LEMMA 4.2. Let $s \geqslant 1$ and $v \in \tilde{H}^{s}$. Then

$$
|(v Z, \rho)|+|(\Lambda Z, \rho)| \leqslant C|| Z\left|\left\|_{-s}|| \rho \mid\right\|_{s}, \quad Z \in \hat{H}_{\mathfrak{p}}^{1}, \rho \in \tilde{H}^{s} .\right.
$$

The constant $C$ depends on $s$ and $v$, but is independent of $\bar{x}$.

Proof. Since $\left\|v \rho\left|\left\|_{s} \leqslant C\left|\left\|v\left|\left\|_{s}\right\| \rho\right|\right\|_{s}\right.\right.\right.\right.$, it suffices to bound $|(v Z, \rho) \mid$ only in case $v=1$. Define $\phi$ as in Lemma 4.1. Then

$$
|(Z, \rho)|=|A(Z, \phi)| \leqslant\left\|Z \left|\left\|_ { - s } \left|\left\|\phi\left|\left\|_{s+2} \leqslant C\right\| Z\right|\right\|_{-s}\|\rho \mid\|_{s} .\right.\right.\right.\right.
$$

Also

$$
|(\Lambda Z, \rho)|=|(Z, \Lambda \rho)| \leqslant C\left|\left\|Z \left|\left\|_ { - s } | | \Lambda \rho \left|\left\|_{s} \leqslant C\left|\left\|Z \left|\left\|_ { - s } \left|\left\|\rho|\||_{s} .\right.\right.\right.\right.\right.\right.\right.\right.\right.\right.\right.\right.
$$

LEMMA 4.3. For all integers $s$ and all $Z \in \hat{H}_{\mathfrak{p}}^{1}$,

$$
|Z(\bar{x})| \leqslant C|| Z \mid \|_{-s} \text {. }
$$

The constant $C$ may be taken to be independent of $\bar{x}$.

Proof. Note that if $s<0$, the desired result holds with $C=1$. Let $G$ be the periodic function defined by

$$
G(x)=[6(x-\bar{x})(x-\bar{x}-1)+1] / 12, \quad \bar{x}<x \leqslant \bar{x}+1 .
$$

Then $G \in\left(\tilde{H}^{s}\right)^{\wedge}$ for all $s \geqslant 0$ and $\|G\|_{s} \leqslant\|G\|_{2}$. It is easily checked that $Z(\bar{x})=A(G, Z)$, and so $|Z(\bar{x})| \leqslant\|G\|_{2}\|\mid Z\|_{-s}$.

We are now ready to analyze the quasiprojection.

Proposition 4.4. Let $s \geqslant-1$ and $m, i \geqslant 0$ be integers such that $s+2 i<r-2$. Then there is a constant $C$, independent of $\bar{x}$, for which

$$
\left\|d_{t}^{m} Z_{i}\right\|_{-s} \leqslant C h^{r+2 i+s}, \quad 0 \leqslant t \leqslant T .
$$

Proof. By induction on $i$. From the definition of $V$ and the approximation properties of the space $S$, we have

$$
\left\|d_{x} d_{t}^{m} Z_{0}\right\|^{2}=\inf _{\mu \in S} A\left(d_{t}^{m} Z_{0}, d_{t}^{m} u-\mu\right)<C\left\|d_{x} d_{t}^{m} Z_{0}\right\| h^{r-1}
$$


Since the definition of the elliptic projection also implies that $\left(d_{t}^{m} Z_{0}, 1\right)=0$, it follows that $\left\|\mid d_{t}^{m} Z_{0}\right\|_{1} \leqslant C h^{r-1}$. To estimate $\left\|d_{t}^{m} Z_{0}\right\|_{-s}, 0 \leqslant s \leqslant r-2$, we consider $\phi \in \tilde{H}^{s+2}$. Then

$$
A\left(d_{t}^{m} Z_{0}, \phi\right)=\inf _{\mu \in S} A\left(d_{t}^{m} Z_{0}, \phi-\mu\right) \leqslant C\left\||| d_{t}^{m} Z_{0}\right\|_{1}\|\phi\|_{s+2}
$$

so $\left\|d_{t}^{m} Z_{0}\right\|_{-s} \leqslant C h^{r+s}$. This proves the proposition in case $i=0$.

Suppose now that $0 \leqslant i \leqslant(r-1) / 2$. By differentiating (3.6) $m$ times with respect to $t$, setting $\mu=d_{t}^{m} Z_{i}$, and invoking the inductive hypothesis, we observe that

$$
\left\|d_{t}^{m} Z_{i}\right\|_{1} \leqslant C \sum_{l=0}^{m+3} \sup _{0<t<T}\left\|d_{t}^{l} Z_{i-1}\right\|_{-1} \leqslant C h^{r+2 i-1} .
$$

To complete the proof we must show that

$$
\left\|d_{t}^{m} Z_{i}\right\|_{-s} \leqslant C h^{r+2 i+s}, \quad 0 \leqslant s \leqslant r-2-2 i .
$$

First let $m>0$. For $\phi \in\left(\tilde{H}^{s+2}\right)^{\wedge}$ with $\|\phi\|_{s+2}=1$,

$$
\begin{aligned}
& A\left(d_{t}^{m} Z_{i}, \phi\right)= \inf _{\mu \in \hat{S}}\left[A\left(d_{t}^{m} Z_{i}, \phi-\mu\right)+\left(\Lambda d_{t}^{m+1} Z_{i-1}+2 d_{t}^{m}\left(u Z_{i-1}\right), \phi\right)\right. \\
&\left.-\left(\Lambda d_{t}^{m+1} Z_{i-1}+2 d_{t}^{m}\left(u Z_{i-1}\right), \phi-\mu\right)\right] \\
& \leqslant C\left(h^{s+1}\left\|d_{t}^{m} Z_{i}\right\|_{1}+\sum_{l=0}^{m+1}\left\|d_{t}^{l} Z_{i-1}\right\|_{-s-2}+h^{s+1} \sum_{l=0}^{m+1}\left\|d_{t}^{l} Z_{i-1}\right\|_{1}\right),
\end{aligned}
$$

which together with the inductive hypothesis yields the desired estimate. In case $m=0$, we obtain the result by essentially the same argument, including one extra term from (3.6).

Recall that $j=[(r-1) / 2]$.

COROllary 4.5. If $r$ is even, then

$$
\sum_{m=0}^{2}\left\|d_{t}^{m} Z_{j}\right\|<C h^{2(r-1)}, \quad 0 \leqslant t \leqslant T .
$$

If $r$ is odd, then

$$
\sum_{m=0}^{2}\left\|d_{t}^{m} Z_{j}\right\|_{1} \leqslant C h^{2(r-1)}, \quad 0 \leqslant t \leqslant T .
$$

Corollary 4.6. For $i=0,1, \ldots, j$,

$$
\left|Z_{i}(\bar{x}, t)\right| \leqslant C\left(k^{2}+h^{2(r-1)}\right), \quad \bar{x} \in \delta, 0 \leqslant t \leqslant T .
$$

Proof. Set $m=0, s=r-2 j-2$ in the proposition and invoke Lemma 4.3.

Corollary 4.7. For $i=1,2, \ldots, j$,

$$
\left\|Z_{i}^{0}\right\|_{1}+\left\|Z_{i}^{1}\right\|_{1}+\left\|\bar{Z}_{i}\right\|_{1} \leqslant C\left(k^{2}+h^{2(r-1)}\right) .
$$

Proof. From (3.6),

$$
\left\|d_{x} Z_{i}^{1}\right\| \leqslant\left\|\Lambda\left(d_{t} Z_{i-1}^{1}-\overline{d_{t} Z_{i-1}}\right)\right\|_{-1}+2\left\|u^{1} Z_{i-1}^{1}-\bar{u} \bar{Z}_{i-1}\right\|_{-1},
$$


so $\left\|Z_{i}^{1}\right\|_{1} \leqslant C k^{3}$. Therefore, $\left\|\bar{Z}_{i}\right\|_{1} \leqslant\left\|Z_{i}^{1}\right\|_{1}+\left\|\bar{Z}_{i}-Z_{i}^{1}\right\|_{1} \leqslant C k^{3}$. Finally,

$$
\left\|Z_{i}^{0}\right\|_{1} \leqslant\left\|Z_{i}^{1}\right\|_{1}+k \sup _{0<t<k}\left\|d_{t} Z_{i}\right\|_{1} \leqslant C\left(k^{2}+h^{2(r-1)}\right)
$$

by the proposition.

COROLlaRY 4.8. The global estimates (3.10) hold.

Proof. It suffices to prove (3.9). From the choices of $U^{0}$ and $U^{1}$ it follows that

$$
\left\|e^{0}\right\|_{1}+\left\|e^{1}\right\|_{1}+\left\|D_{+} e^{0}\right\|_{1} \leqslant C\left(k^{2}+h^{r-1}\right) \text {. }
$$

By (3.7) and (2.7), we have

$$
D e^{1}=\left(D u^{1}-D V^{1}\right)+\left(D V^{1}-\bar{V}_{t}\right)+\sum_{i=1}^{j} \overline{d_{t} Z_{i}},
$$

and hence, by Proposition 4.4, $\left\|D e^{1}\right\| \leqslant C\left(k^{2}+h^{r-1}\right)$. Therefore, $D_{+} e^{1}=2 D e^{1}-$ $D_{+} e^{0}$ is of the same order.

It remains to bound $\|\varepsilon\|_{-1}$. From (2.1) (with $\phi=\varepsilon$ ), (3.8), and the proposition it follows that $\|\varepsilon\|_{-1} \leqslant C\left(k^{2}+h^{r-1}\right)$.

Before proceeding to the proof of the superconvergence theorem, we prove one final lemma.

LEMMA 4.9. For each $\eta>0$ there exists $h_{\eta}>0$ such that for $h \leqslant h_{\eta}$ and $\rho \in \hat{H}_{\mathfrak{p}}^{1}$

$$
\|\rho\|^{2} \leqslant \eta\left\|\rho_{x}\right\|^{2}+\frac{1}{2 \eta}\left\|P_{0} \Lambda \rho\right\|^{2} .
$$

Proof. Clearly

$$
\|\rho\|^{2}=-\left(\Lambda \rho, \rho_{x}\right) \leqslant \frac{\eta}{2}\left\|\rho_{x}\right\|^{2}+\frac{1}{2 \eta}\|\Lambda \rho\|^{2}
$$

Now

$$
\|\Lambda \rho\|^{2}=\left\|P_{0} \Lambda \rho\right\|^{2}+\left\|\left(P_{0}-I\right) \Lambda \rho\right\|^{2} \leqslant\left\|P_{0} \Lambda \rho\right\|^{2}+C h^{4}\left\|\rho_{x}\right\|^{2} .
$$

The lemma foliows with $h_{\eta}=(\eta / 2 C)^{1 / 4}$.

We now turn to the proof of the superconvergence theorem. Setting $\theta_{0}=V-U$ and $\theta=\theta_{0}-\sum_{i=1}^{j} Z_{i}$, we shall employ the decomposition of the error $e=\theta+$ $\sum_{i=0}^{j} Z_{i}$. In light of Corollary 4.6, it suffices to prove that

$$
\|\theta\|_{1} \leqslant C\left(k^{2}+h^{2(r-1)}\right), \quad n=0,1, \ldots, N .
$$

This we shall accomplish by the method of energy estimates.

From the Korteweg-de Vries equation and the definition of the elliptic projection we have

$$
\begin{aligned}
-\left(\Lambda D V+(u)^{2}, \mu\right)+A(\tilde{V}, \mu) & \\
& =\left(\Lambda D Z_{0}-\Lambda\left(D u-\tilde{u}_{t}\right)+\widetilde{(u)^{2}}-(u)^{2}, \mu\right), \quad \mu \in \hat{S} .
\end{aligned}
$$

Comparing this equation with (2.5), we see that

$$
\text { (4.4) } B\left(\theta_{0}, \mu\right)=\left(J Z_{0}-\varepsilon-\Lambda\left(D u-\tilde{u}_{t}\right)+\widetilde{(u)^{2}}-(u)^{2}-(e)^{2}, \mu\right), \quad \mu \in \hat{S} \text {, }
$$
where $J \phi=\Lambda D \phi+2 u \phi$ and $B(\phi, \mu)=-(J \phi, \mu)+A(\tilde{\phi}, \mu)$. Note that the linear operator $J$ and bilinear form $B$ are time-dependent. 
The quasiprojection was constructed so as to cancel those terms of magnitude greater than $O\left(k^{2}+h^{2(r-1)}\right)$ entering into the right-hand side of (4.4). Indeed we may rewrite (3.6) as

$$
\begin{aligned}
& B\left(Z_{i}, \mu\right)=-\left(J\left(Z_{i}-Z_{i-1}\right)+\Lambda \overline{d_{t} Z_{i-1}}+2 \bar{u} \bar{Z}_{i-1}\right. \\
& \left.\quad+\Lambda\left(D Z_{i-1}-\widetilde{d_{t} Z_{i-1}}\right)+2\left(u Z_{i-1}-\widetilde{u Z_{i-1}}\right), \mu\right), \quad \mu \in \hat{S} .
\end{aligned}
$$

Therefore,

$$
\begin{aligned}
\sum_{i=1}^{j} B\left(Z_{i}, \mu\right)= & -\left(J\left(Z_{j}-Z_{0}\right), \mu\right)-\sum_{i=0}^{j-1}\left(\Lambda \overline{d_{t} Z_{i}}+2 \bar{u} \bar{Z}_{i}, \mu\right) \\
& -\sum_{i=0}^{j-1}\left(\Lambda\left(D Z_{i}-\widetilde{d_{t} Z_{i}}\right)+2\left(u Z_{i}-\widetilde{u Z_{i}}\right), \mu\right), \quad \mu \in \hat{S} .
\end{aligned}
$$

Combining this equation with (4.4) and (3.8), we have

$$
B(\theta, \mu)=(Q, \mu), \quad n=1,2, \ldots, N-1,
$$

where $Q^{n} \in \hat{S}$ is defined for $1 \leqslant n \leqslant N-1$ by

$$
\begin{aligned}
& (Q, \mu)=\left(J Z_{j}-\Lambda \overline{d_{t} Z_{j}}-2 \bar{u} \bar{Z}_{j}-\Lambda\left(D u-\bar{u}_{t}\right)+\widetilde{(u)^{2}}-(u)^{2}-(e)^{2}\right. \\
& \quad+\sum_{i=0}^{j-1}\left[\Lambda\left(D Z_{i}-\widetilde{d_{t} Z_{i}}\right)+2\left(u Z_{i}-\widetilde{u Z_{i}}\right)\right]-2\left(u^{1}+\bar{Z}_{0}\right)\left(u^{1}-\bar{u}\right) \\
& \left.\quad+2 \bar{u} \sum_{i=1}^{j} \bar{Z}_{i}-\Lambda\left(u_{t}^{1}-\bar{u}_{t}\right)+d_{x}^{2}\left(u^{0}+k \bar{u}_{t}-u^{1}\right)+\left(e^{1}\right)^{2}, \mu\right) \\
& \quad-k \sum_{i=1}^{j} A\left(\overline{d_{t} Z_{i}}, \mu\right), \quad \mu \in \hat{S} .
\end{aligned}
$$

Using Proposition 4.4, Corollaries 4.5, 4.7, and 4.8, and (2.1), we have

$$
\max _{1<n<N-1}\left\|Q^{n}\right\|_{-1}+\max _{2<n<N-2}\left\|D Q^{n}\right\|_{-1} \leqslant C\left(k^{2}+h^{2(r-1)}\right) .
$$

Note also that $\theta^{0}=-\sum_{i=1}^{n} Z_{i}^{0}$ and $\theta^{1}=V^{1}-\bar{V}-\sum_{i=1}^{j} Z_{i}^{1}$, so (4.3) will be established if we show that

(4.6) $\max _{0<n<N}\left\|\theta^{n}\right\|_{1} \leqslant C\left(\left\|\theta^{0}\right\|_{1}+\left\|\theta^{1}\right\|_{1}+\max _{1<n<N-1}\left\|Q^{n}\right\|_{-1}+\max _{2<n<N-2}\left\|D Q^{n}\right\|_{-1}\right)$.

Setting

$$
\Xi^{m}=\left\|\theta^{0}\right\|_{1}^{2}+\left\|\theta^{1}\right\|_{1}^{2}+\max _{1<n<N-1}\left\|Q^{n}\right\|_{-1}^{2}+\max _{2<n<N-2}\left\|D Q^{n}\right\|_{-1}^{2}+k \sum_{n=0}^{m}\left\|\theta^{n}\right\|_{1}^{2},
$$

we may further reduce to proving that

$$
\left\|\theta^{m}\right\|_{1}^{2} \leqslant \Xi^{m}, \quad m=2,3, \ldots, N .
$$

For, by the discrete form of Gronwall's lemma, (4.7) implies (4.6). Finally, by Lemma 4.9, (4.7) reduces to the two estimates

$$
\begin{aligned}
\left\|d_{x} \theta^{m}\right\|^{2}+\left\|d_{x} \theta^{m+1}\right\|^{2} \leqslant C\left(\Xi^{m+1}+\left\|\theta^{m}\right\|^{2}+\left\|\theta^{m+1}\right\|^{2}\right) & \\
& m=1,2, \ldots, N-1,
\end{aligned}
$$


and

$$
\left\|P_{0} \Lambda \theta^{m}\right\|^{2}+\left\|P_{0} \Lambda \theta^{m+1}\right\|^{2} \leqslant C \Xi^{m+1}, \quad m=1,2, \ldots, N-1 .
$$

Each of these inequalities will follow from an appropriate choice of $\mu$ in (4.5).

First, let $\mu=D \theta^{n}$, giving

$$
\begin{aligned}
\left\|d_{x} \theta^{n+1}\right\|^{2}-\left\|d_{x} \theta^{n-1}\right\|^{2}=8 k\left(u^{n} \theta^{n}, D \theta^{n}\right)+4 k\left(Q^{n}, D \theta^{n}\right), \\
n=1,2, \ldots, N-1 .
\end{aligned}
$$

Sum this equation as $n$ ranges from 1 to $m, m<N$, using the summation by parts formulas

$$
\begin{aligned}
2 k \sum_{n=1}^{m}\left(Q^{n}, D \theta^{n}\right)= & -2 k \sum_{n=2}^{m-1}\left(D Q^{n}, \theta^{n}\right)+\left(Q^{m}, \theta^{m+1}\right) \\
& +\left(Q^{m-1}, \theta^{m}\right)-\left(Q^{1}, \theta^{0}\right)-\left(Q^{2}, \theta^{1}\right)
\end{aligned}
$$

and

$$
2 k \sum_{n=1}^{m}\left(u^{n} \theta^{n}, D \theta^{n}\right)=-k \sum_{n=1}^{m-1}\left(D_{+} u^{n}, \theta^{n} \theta^{n+1}\right)+\left(u^{m}, \theta^{m} \theta^{m+1}\right)-\left(u^{1}, \theta^{0} \theta^{1}\right) .
$$

Since $u^{n}$ and $D_{+} u^{n}$ are bounded, (4.8) follows easily. To prove (4.9), set $\mu=P_{0} \Lambda \tilde{\theta}^{n}$ in (4.5), getting

$$
\begin{aligned}
& \left\|P_{0} \Lambda \theta^{n+1}\right\|^{2}-\left\|P_{0} \Lambda \theta^{n-1}\right\|^{2} \\
& \quad=4 k\left[-\left(2 u^{n} \theta^{n}, P_{0} \Lambda \tilde{\theta}^{n}\right)+A\left(\tilde{\theta}^{n}, P_{0} \Lambda \tilde{\theta}^{n}\right)-\left(Q^{n}, P_{0} \Lambda \tilde{\theta}^{n}\right)\right] .
\end{aligned}
$$

Again, this equation is summed over $n=1,2, \ldots, m<N$. Because we have assumed that $P_{0}$ is bounded in $H^{1}$, the inner products may be bounded in a straightforward manner to yield (4.9). This completes the proof of the superconvergence theorem.

5. Implementation. In this section we discuss the implementation of the proposed method. The key steps are summarized at the end of the section.

An efficient implementation should be based on (2.3). Except in the case $r=2$, the spaces $S$ and $S^{*}$ have local bases with elements supported in at most two subintervals, and so the coefficient matrix indicated by (2.3) will have periodic banded structure. This matrix is independent of the timestep. (In case $r=2, S^{*}$ has a basis consisting of quadratic $B$-splines with supports in three subintervals.) The equivalent form of the method given by (2.5) should be avoided in practice since, with a usual choice of basis, it leads to a full matrix problem.

From (2.3) we see that in addition to $U^{0}=V^{0}$ and $U^{1}=\bar{V}$, the quantity $\left(\varepsilon, \chi_{x}\right)$ must be computed for all $\chi$ in a basis for $S^{*}$. This is accomplished by first computing the function $W$ given by (3.7) and then using (2.6).

We shall sketch a practical procedure for the computation of $U^{0}, U^{1}$, and $\left(\varepsilon, \chi_{x}\right)$. For simplicity we assume $j=1$, i.e., that the trial functions are quadratic or cubic. The procedure in case of higher-order elements follows along similar lines.

The initial profile is given. From the equations

$$
\begin{aligned}
u_{t} & =-2 u u_{x}-u_{x x x}, \\
u_{t t} & =-2\left(u u_{t}\right)_{x}-u_{t x x x}, \\
u_{t t t} & =-2\left(u_{t} u_{t}+u u_{t t}\right)_{x}-u_{t t x x x}, \\
u_{t t t t} & =-2\left(3 u_{t} u_{t t}+u u_{t t t}\right)_{x}-u_{t t t x x x},
\end{aligned}
$$


$d_{t}^{m} u^{0}$ is determined for $m \leqslant 4$. Next $d_{t}^{m} V^{0} \in \hat{S}+\left(d_{t}^{m} u^{0}, 1\right)$ must be determined satisfying the equations

$$
A\left(d_{t}^{m} V^{0}, \mu\right)=A\left(d_{t}^{m} u^{0}, \mu\right), \quad \mu \in \hat{S} .
$$

It is suggested that $d_{t}^{m} V^{0}$ be computed as $\psi-(\psi, 1)+\left(d_{t}^{m} u^{0}, 1\right)$, where $\psi \in S_{0}=$ $\{\chi \in S \mid \chi(0)=0\}$ is determined by

$$
A(\psi, \mu)=A\left(d_{t}^{m} u^{0}, \mu\right), \quad \mu \in S_{0} .
$$

The space $S_{0}$ is preferred to $\hat{S}$ since it has a local basis consisting of functions supported in only two adjacent subintervals on $[0,1]$. The matrix is thus banded (without corner elements such as arise in periodic problems). Its factorization should be computed and stored as it will be used several times.

Once the functions $d_{t}^{m} V^{0}, m \leqslant 4$, are computed, then $U^{0}, U^{1}$, and also $d_{t}^{m} Z_{0}^{0}$ are known. Next $d_{t}^{m} Z_{1}^{0}, m \leqslant 3$, must be computed using the defining equation (3.6). Differentiating this equation $m$ times and setting $t=0$ yields an equation for $\phi=d_{t}^{m} Z_{1}^{0}$ of the form

$$
A(\phi, \mu)=(f, \mu), \quad \mu \in \hat{S} .
$$

Here the unknown function $\phi$ is sought in $\hat{S}$, and $f \in H^{0}$ is known. As above, one can avoid the space $\hat{S}$ in favor of $S_{0}$ by computing $\psi \in S_{0}$ such that

$$
A(\psi, \mu)=(f-(f, 1), \mu), \quad \mu \in S_{0},
$$

and setting $\phi=\psi-(\psi, 1)$. Since every function in $S$ differs from a function in $S_{0}$ by a constant, it follows that $A(\phi, \mu)=A(\psi, \mu)=(f-(f, 1), \mu)$ for all $\mu \in S$, whence (5.3).

Finally, it is now straightforward to compute the necessary inner products $\left(\varepsilon, \chi_{x}\right)$ from (2.6) and (3.7).

For convenient reference we list here the major steps of the computation.

1. For $m=0,1, \ldots, 4$ :

1.1 solve (5.2) for $\psi \in S_{0}$;

1.2 set $d_{t}^{m} V^{0}=\psi-(\psi, 1)+\left(d_{t}^{m} u^{0}, 1\right)$;

1.3 set $d_{t}^{m} Z_{0}^{0}=d_{t}^{m} u^{0}-d_{t}^{m} V^{0}$.

2. Set $U^{0}=V^{0}, U^{1}=\bar{V}$.

3. Find $\psi \in S_{0}$ satisfying

$$
\begin{aligned}
A(\psi, \mu)= & \left(\left[\Lambda d_{t} Z_{0}^{0}+2 u^{0} Z_{0}^{0}\right]-\left[\Lambda \overline{d_{t} Z_{0}}+2 \bar{u} \bar{Z}_{0}\right], \mu\right) \\
& -2\left(u^{0} Z_{0}^{1}-\bar{u} \bar{Z}_{0}, 1\right)(\mu, 1), \quad \mu \in S_{0}
\end{aligned}
$$

4. Set $Z_{1}^{0}=\psi-(\psi, 1)$.

5. For $m=1,2,3$ :

5.1 find $\psi \in S_{0}$ satisfying

$$
\begin{aligned}
A(\psi, \mu)= & \left(\left[\Lambda d_{t}^{m+1} Z_{0}^{0}+2 d_{t}^{m}\left(u Z_{0}\right)^{0}\right], \mu\right) \\
& -2\left(d_{t}^{m}\left(u Z_{0}\right)^{0}, 1\right)(\mu, 1), \quad \mu \in S_{0} ;
\end{aligned}
$$

5.2 set $d_{t}^{m} Z_{1}^{0}=\psi-(\psi, 1)$.

6. Set $W=\bar{V}_{t}-\overline{d_{t} Z_{1}}$.

7. Set $\left(\varepsilon, \chi_{x}\right)=-\left(\Lambda W+\left(U^{1}\right)^{2}, \chi_{x}\right)+A\left(U^{0}+k W, \chi_{x}\right), \chi \in S^{*}$.

8. For $n=1,2, \ldots, N-1$ define $U^{n+1} \in \hat{S}+\left(u_{0}, 1\right)$ by (2.3). 
6. Errors in the Data. In the proof of the superconvergence theorem we have assumed that the quantities $U^{0}, U^{1}$, and $W$ entering into the scheme given by (2.3) and (2.6) were taken to be $V^{0}, \bar{V}$, and $\bar{V}_{t}-\sum_{i=1}^{j} \bar{d}_{t} Z_{i}$, respectively. In fact, it is sufficient that these values be approximated to order $k^{2}+h^{2(r-1)}$, as is sketched in the final paragraph.

Let $W_{0}=\bar{V}_{t}-\sum_{i=1}^{j} \bar{d}_{t} Z_{i}$, and let $\varepsilon_{0}$ be the corresponding solution of (2.6), i.e.,

$$
\left(\varepsilon_{0}, \mu\right)=-\left(\Lambda W_{0}+\left(U^{1}\right)^{2}, \mu\right)+A\left(U^{0}+k W_{0}, \mu\right), \quad \mu \in S .
$$

Consider the method given by (2.3) and (2.6) with $U^{0}, U^{1} \in \hat{S}+\left(u_{0}, 1\right)$ and $W \in \hat{S}$ arbitrary. Supposing that the quantities $\left\|U^{0}-V^{0}\right\|_{1},\left\|U^{1}-\bar{V}\right\|_{1}$, and $h$ are sufficiently small, minor modifications to the argument of Section 4 yield the estimate on the error $e^{n}=u^{n}-U^{n}$

$$
\begin{aligned}
\left|e^{n}(\bar{x})\right| \leqslant C\left\{k^{2}+h^{2(r-1)}+\left\|U^{0}-V^{0}\right\|_{1}+\left\|U^{1}-\bar{V}\right\|_{1}\right. & \\
+\left\|\varepsilon-\varepsilon_{0}\right\|_{-1}+\| W- & \left.W_{0}\left\|_{1}^{2}+\right\| D_{+} e^{0} \|_{1}^{2}\right\}, \\
& \bar{x} \in \delta, n=0,1, \ldots, N .
\end{aligned}
$$

Also from (2.1), (2.6), and (5.1) we obtain

$$
\left\|\varepsilon-\varepsilon_{0}\right\|_{-1} \leqslant C\left\{\left\|W-W_{0}\right\|_{-2}+k\left\|W-W_{0}\right\|_{1}\right\} .
$$

It follows that if $U^{0}-V^{0}, U^{1}-\bar{V}$, and $W-W_{0}$ are $O\left(k^{2}+h^{2(r-1)}\right)$ in appropriate norms (e.g., in $H^{1}$ ), then the superconvergence theorem stated at the end of Section 3 holds.

\author{
Department of Mathematics and \\ Institute for Physical Science and Technology \\ University of Maryland \\ College Park, Maryland 20742 \\ Østfold Distriktshбgskole \\ 1750 Halden, Norway
}

1. D. N. ARnOLd \& J. Douglas, JR., "Superconvergence of the Galerkin approximation of a quasilinear parabolic equation in a single space variable," Calcolo, v. 16, 1979, pp. 345-369.

2. D. N. ARnold, J. Douglas, JR. \& V. Thomée, "Superconvergence of a finite element approximation to the solution of a Sobolev equation in a single space variable," Math. Comp., v. 36, 1981, pp. 53-63.

3. T. B. Benjamin, J. L. Bona \& J. J. Mahony, "Model equations for long waves in nonlinear dispersive systems,” Philos. Trans. Roy. Soc. London Ser. A, v. 272, 1972, pp. 47-78.

4. J. L. Bona \& R. SMrth, "The initial-value problem for the Korteweg-de Vries equation," Philos. Trans. Roy. Soc. London Ser. A, v. 278, 1975, pp. 555-601.

5. J. Douglas, JR., T. DUPONT \& M. F. WheELER, “A quasi-projection analysis of Galerkin methods for parabolic and hyperbolic equations," Math. Comp., v. 32, 1978, pp. 345-362.

6. G. B. Whтtнам, Linear and Nonlinear Waves, Wiley, New York, 1974.

7. R. WINTHER, “A conservative finite element method for the Korteweg-de Vries equation," Math. Comp., v. 34, 1980, pp. 23-43. 